\title{
Resistência mecânica à adesão em superfícies de madeira de pinus aplainadas e unidas por adesivos PVAc
}

\section{Mechanical adhesion strength on pine wood surfaces planed and bonded by PVAc adhesives}

\author{
João Carlos Biazzon ${ }^{1}$, Victor Almeida De Araujo ${ }^{2}$, \\ Paulo Roberto Gomes Alves ${ }^{3}$, Elen Aparecida Martines Morales ${ }^{3}$, \\ Marcos Tadeu Tibúrcio Gonçalves ${ }^{3}$, Ivaldo De Domenico Valarelli ${ }^{1}$
}

\footnotetext{
${ }^{1}$ Departamento de Engenharia Mecânica, Universidade Estadual Paulista - UNESP, Campus de Bauru. Avenida Luiz Edmundo Carrijo Coube, 14-01, Caixa Postal 473, Bauru, São Paulo, Brasil.

${ }^{2}$ Departamento de Ciências Florestais, Universidade de São Paulo - USP-ESALQ, Escola Superior de Agricultura Luiz de Queiroz. Avenida Padua Dias, 11, 13418-900, Piracicaba, São Paulo, Brasil.

${ }^{3}$ Curso de Engenharia Industrial Madeireira, Universidade Estadual Paulista - UNESP. Campus de Itapeva. Rua Geraldo Alckmin, 519, 18409-010, Itapeva, São Paulo, Brasil

e-mail: jcbiazzon@gmail.com, engim.victor@yahoo.de,gomespaulo13@yahoo.com.br, elen@itapeva.unesp.br, marcostadeu@itapeva.unesp.br, ivaldo@feb.unesp.br.
}

\section{RESUMO}

Este trabalho teve como objetivo analisar o desempenho de juntas coladas de madeiras da espécie Pinus elliottii com a utilização de dois adesivos comerciais à base de poliacetato de vinila (PVAc), com diferentes características físicas e composição química, ambos testados nas formas diluída em água e não diluída. As superfícies dos corpos de prova de madeira foram usinadas em processo de fresamento discordante. Uma plaina desengrossadeira de madeira com cabeçote de quatro facas afiadas de aço carbono semi-rápido à base de $\mathrm{Cr}$ $\mathrm{Ni}-\mathrm{V}$ foi utilizada. Essa máquina foi preparada para atuar com velocidade de corte $\left(\mathrm{V}_{\mathrm{c}}\right)$ de $30,18 \mathrm{~m} . \mathrm{s}^{-1}$ e velocidade de avanço da madeira $\left(V_{f}\right)$ de $11,75 \mathrm{~m} \cdot \mathrm{min}^{-1}$, resultando num avanço por dente $\left(\mathrm{f}_{\mathrm{z}}\right)$ de $0,56 \mathrm{~mm}$. Os corpos de prova foram confeccionados para ensaios de resistência a ruptura na linha de cola por esforços solicitantes ao cisalhamento de acordo com a ABNT NBR 7190:1997. Para a confecção dos corpos de prova, as peças foram coladas de maneira aleatória em relação aos planos de formação dos anéis de crescimento da madeira. Os seguintes valores médios de resistência ao cisalhamento na linha de cola $\left(f_{v 0}\right)$, na forma diluída e não diluída foram, respectivamente, PVAc-M: 6,52 e 6,49 MPa; PVAc-H: 5,86 e 5,15 MPa. Em relação à resistência ao cisalhamento na linha de cola, concluiu-se que ambos adesivos na forma diluída com 33,33\% de água resultaram em maior adesão em relação à formulação original, sendo que o adesivo diluído do tipo $\mathrm{M}$ proporcionou um aumento de $21,01 \%$ na resistência à ruptura em relação ao adesivo não diluido do tipo "H".

Palavras-chave: linha de cola, resistência ao cisalhamento, usinagem, madeira.

\section{ABSTRACT}

This study aimed to analyze the performance of bonded joints of the Pinus elliottii wood species with the use of two commercial adhesives based on vinyl polyacetate (PVAc) with different physical characteristics and chemical composition, both tested on water diluted and undiluted modes. The surfaces of wood specimens were machined in discordant milling process. A wood thickness planer with headstock of four semi-fast carbon steel sharpened knives based on $\mathrm{Cr}-\mathrm{Ni}-\mathrm{V}$ was used. This machine was prepared to operate with cutting speed $\left(V_{c}\right)$ of $30.18 \mathrm{~ms}^{-1}$ and feed rate of wood $\left(V_{f}\right)$ of $11.75 \mathrm{~m} \cdot \mathrm{min}^{-1}$, resulting in an feed rate per tooth $\left(\mathrm{f}_{\mathrm{z}}\right)$ of $0.56 \mathrm{~mm}$. The specimens were prepared for tests of resistance to rupture in the glue line by the shear stress efforts according to the ABNT NBR 7190:1997 standard. In order to specimen preparation, the parts were randomly glued in relation to the formation plans of the wood growth rings. The following average values of shear strength in the glue line $\left(f_{v 0}\right)$ obtained in diluted and undiluted forms were, respectively, PVAc - M: 6.52 and 6.49 MPa; PVAc - H: 5.86 and 5.15 MPa. In relation to the shear stress at the glue line, it was concluded that both adhesives in diluted form with $33.33 \%$ of water resulted in higher adhesion strength in com- 
parison to the original formulation, whereas the diluted adhesive type M provided an increasing of $21.01 \%$ regarding the undiluted adhesive of type $\mathrm{H}$.

Keywords: glue line, shear strength, machining, wood.

\section{INTRODUÇÃO}

A madeira é uma matéria-prima indispensável em aplicações na construção civil, indústria moveleira, indústria de papel e celulose, dentre outras. Na sua forma maciça e em dimensões maiores, a madeira tornou-se, com o passar dos anos, um material difícil de ser encontrado devido à constante diminuição de florestas nativas e à legislação ambiental vigente. Assim, os setores que a utilizam buscaram novas alternativas para um aproveitamento mais racional [1].

Apesar de o Brasil possuir uma vasta área de plantio de reflorestamento, com o passar dos anos, percebeu-se a necessidade cada vez maior de racionalização do processo de usinagem da madeira, principalmente através do desenvolvimento de novas tecnologias, que além de diminuírem a geração de resíduos com a melhor utilização dos recursos florestais, também melhoram algumas de suas características [2].

Souza et al. [3] relatam que a usinagem está presente na fabricação de diversos produtos que são industrializados no setor madeireiro, sendo que, dentro do seu processo, em algum momento ou etapa de produção, a madeira será usinada, e também mencionam que é necessário a compreensão dos conceitos desses processos e das suas variáveis, para obter uma melhor visão de mercado e qualidade dos produtos dentro do âmbito industrial.

No fresamento convencional ou discordante (up-milling) as ferramentas movem-se contrariamente ao movimento da peça; enquanto que no fresamento concordante (dow-milling) a ferramenta e a peça se movimentam na mesma direção. Esse tipo de classificação somente vale no fresamento cilíndrico tangencial, de grande utilização no beneficiamento de madeiras [4].

Há vários fatores da madeira que podem influenciar na colagem. Frihart e Hunt [5] relatam sobre a importância da superfície das peças de madeira, que deve ser lisa, aplainada e livre de marcas de usinagem ou outras irregularidades, incluindo "batidas" de plaina, partes esmagadas, farpas e fibras soltas. Portanto, as condições de acabamento das superfícies são extremamente importantes para um resultado satisfatório, pois a colagem por adesivos consiste na união de superfícies.

De acordo com Kollmann et al. [6] e Kollmann e Côté [7], outros fatores que influenciam na colagem da madeira são as suas propriedades anatômicas, físicas, mecânicas e químicas (extrativos e pH).

No processo de colagem há dois fenômenos físico-químicos: a adesão e a coesão [8]. A adesão é a resistência mecânica da interface entre o filme adesivo e o substrato enquanto que a coesão é a resistência interna do filme adesivo através de forças de valência primárias ou secundárias.

A força coesiva de um adesivo polimérico depende do tamanho molecular, da organização macromolecular e da uniformidade supermolecular [9].

Segundo Pizzi [10], as principais teorias existentes sobre adesão na madeira apresentadas na literatura são: Mecânica, Adsorção, Difusão e Adesão Química.

Kollmann et al. [6] acreditam que a combinação entre as teorias de adesão é o que mais se aproxima da realidade. Schultz e Nardin [11] enfatizam que os modelos teóricos que têm sido propostos, juntos, são complementares e contraditórios, mas não se anulam e dependem em grande parte do sistema escolhido. Cada uma destas teorias é válida, em certa medida, dependendo da natureza dos sólidos em contato e com as condições de formação do sistema ligado. Também mencionam que normalmente esses modelos se distinguem entre mecânica e adesão específica, sendo esta última calculada com base nos vários tipos de ligações (eletrostática, secundária, primária) que podem ocorrer entre dois sólidos.

Segundo Jakes et al. [12] e Follrich et al. [13], a penetração do adesivo na estrutura porosa da madeira pode ocorrer na escala micrométrica e que, nesta escala, a penetração do adesivo ocorre na célula lumina, ou seja, no lúmen, e na escala nanométrica a penetração do adesivo ocorre na parede celular, Cool e Hernández [14] também corroboram com esta ideia.

Outros fatores que também podem apresentar efeitos sobre o resultado de adesão são: o espalhamento do adesivo, a pressão aplicada e as propriedades de superfície resultantes de processos de usinagem incluindo limpeza da superfície, danos estruturais e rugosidade [13].

Segundo Frihart [15] o poliacetato de vinila (PVAc) e seus derivados atualmente são os adesivos mais usados pelos diversos segmentos da indústria moveleira e/ou madeireira para aplicações de uso interior.

Petrie [16] e Salvini et al. [17] mencionam que o PVAc é a mais versátil das resinas vinílicas, geral- 
mente disponíveis em soluções ou emulsões, podendo ser são ou não plastificadas.

Santos [18] relata que os adesivos de PVAc possuem uma boa adesão a superfícies celulósicas tais como madeira e papel.

Segundo Mesquita [19] o PVAc é um polímero termoplástico, amorfo e atático (grupos laterais estão distribuídos aleatoriamente e ramificados), e algumas de suas propriedades dependem intrinsicamente da massa molar, alterando suas propriedades como por exemplo: a solubilidade, a densidade e o ponto de amolecimento, podendo variar de líquidos viscosos a sólidos.

Para Kim e Kim [20] há um maior interesse em adesivos à base de PVAc por esse apresentar uma maior força vinculativa e melhores propriedades de filme e tem crescido consideravelmente nas últimas duas décadas, devido à sua ampla utilização em adesivos.

Por serem à base de água, os adesivos de PVAc apresentam vantagens como: aplicação simples, têem baixa toxicidade, apresentam baixo custo se comparados a outras resinas comerciais, possuem baixa inflamabilidade, sob condições adequadas secam rápido, são de fácil limpeza e possuem boa estabilidade à estocagem [21].

Kim e Kim [20], Özçifçi e Yapıc1 [22] relatam que o PVAc é de fácil aplicação e não danifica as ferramentas durante o processo de corte. No entanto, a resistência mecânica de PVAc diminui com o aumento da temperatura, perdendo a capacidade de resistência de ligação acima de $70^{\circ} \mathrm{C}$.

Segundinho et al. [1] utilizaram vários adesivos, dentre eles o PVAc, o qual apresentou bons resultados nos ensaios de cisalhamento e delaminação, devido à maior penetração do adesivo na madeira (interface madeira-adesivo).

O presente trabalho teve por objetivo avaliar a qualidade de colagem da madeira serrada da espécie Pinus elliottii, em testes de resistência na linha de cola em ensaios de cisalhamento, a fim de qualificar as interações madeira/adesivo. Foram utilizados dois adesivos diferentes de PVAc, disponíveis no mercado, na forma diluída e não diluída, empregando-se velocidade de avanço de $11,57 \mathrm{~m} \cdot \mathrm{min}^{-1}$ na geração das superfícies com planos aleatórios de colagem, com relação à disposição dos anéis de crescimento.

\section{MATERIAIS E MÉTODOS}

\subsection{Preparação de amostras}

A madeira usada para este estudo foi o Pinus elliottii devido as suas características gerais: baixa densidade, é uma madeira macia ao corte, possui grã direita e textura fina, apresenta boa trabalhabilidade e possui um amplo espectro de uso como, por exemplo, na construção civil, mobiliário, embalagens, etc. [23].

Foi escolhida uma prancha de pinus na serraria da UNESP de Itapeva a qual foi desengrossada (desbastada) para retirar as irregularidades. Depois de ser usinada em plainas para a regularização de superfícies e normalização das dimensões das partes, a prancha foi cortada em sarrafos ficando com as dimensões nominais $55 \times 33 \times 650 \mathrm{~mm}$.

Ao término desse desdobramento, a madeira foi levada para a sala climatizada a $12^{\circ} \mathrm{C}$, com ar condicionado a $20^{\circ} \mathrm{C}$ e desumidificador a $60 \%$ de umidade relativa, para as peças atingirem a umidade de $12 \%$. Estabilizados os sarrafos, foram aplainados com velocidade de avanço de $11,57 \mathrm{~m} \cdot \mathrm{min}^{-1}$ resultando em um avanço por dente " $\mathrm{f}_{\mathrm{z}}$ " da ferramenta de corte de $0,56 \mathrm{~mm}$, até atingir as espessuras de $20 \mathrm{~mm}$ e $30 \mathrm{~mm}$. Terminando o processo de usinagem, as partes ficaram com as dimensões nominais $55 \times 20 \times 650 \mathrm{~mm} \mathrm{e}$ $55 \times 30 \times 650 \mathrm{~mm}$.

Na preparação das superfícies para aplicação dos adesivos, as amostras de madeira foram selecionadas e usinadas por operação de fresamento cilíndrico tangencial e coladas de maneira aleatória em relação aos planos de formação dos anéis de crescimento da madeira. A gramatura utilizada para ambos adesivos diluídos e não diluídos foi de $200 \mathrm{~g} \cdot \mathrm{m}^{-2}$.

Após a colagem, as peças usinadas foram prensadas a frio com uma pressão de $6 \mathrm{Kgf.cm}^{-2}$ (0,59 $\mathrm{MPa}$ ), com o tempo de prensagem de 4 horas, em uma prensa hidráulica.

As amostras foram preparadas em quatro grupos de ensaios em relação ao tipo do adesivo aplicado sobre as superfícies de colagem, da seguinte forma: HP - Henkel ${ }^{\circledR}$ não diluído; HD - Henkel ${ }^{\circledR}$ diluído; MP Madeiranit $^{\circledR}$ não diluído e MD - Madeiranit ${ }^{\circledR}$ diluído, classificados como D1 de acordo com a EN 204 (2001) e EN 205 (2001). 


\subsection{Adesivos}

Para os ensaios de resistência mecânica na ruptura na linha de cola as amostras foram coladas com dois tipos de adesivos à base de poliacetato de vinila denominados neste estudo por "PVAc - Henkel $^{\circledR}(\mathrm{PVAc}-\mathrm{H})$ " $\mathrm{e}$ "PVAc - Madeiranit ${ }^{\circledR}$ (PVAc - M)". Esses adesivos foram testados em duas condições: diluído e não diluído.

Os adesivos foram diluídos em água deionizada, nas proporções 2:1, v/v, de Adesivo: $\mathrm{H}_{2} \mathrm{O}$ (volume de adesivo em relação ao volume de água), ou seja, a cada $2 \mathrm{~mL}$ de adesivo foi adicionado $1 \mathrm{~mL} \mathrm{de} \mathrm{H}_{2} \mathrm{O}$, originando uma diluição de 33,33\%.

As viscosidades dos adesivos foram determinadas através do método do copo Ford NBR 5849:2015, ASTM-D 1200:2014, o qual mede o tempo de escoamento em segundos. Segundo o fabricante do viscosímetro, o tempo final de escoamento do material deve ser realizado na primeira quebra do escoamento contínuo. O experimento foi realizado em triplicata para obter a viscosidade média.

A viscosidade em centistokes é determinada pela Equação 1, conforme a indicação do fabricante.

$$
V=3,85(t-4,49)
$$

A Tabela 1 apresenta os valores médios da viscosidade dos adesivos não diluídos e diluídos em Centistokes (cSt) e Centipoises (cP).

Tabela 1: Valores médios da viscosidade dos adesivos.

\begin{tabular}{lll}
\hline ADESIVOS & VISCOSIDADE (cSt) & VISCOSIDADE (cP) \\
\hline Madeiranit $^{\circledR}$ não diluído (MP) & 91,130 & 10147,27 \\
\hline Madeiranit $^{\circledR}$ diluído (MD) & 65,450 & 7107,87 \\
Henkel $^{\circledR}$ não diluído (HP) & 69,069 & 7963,65 \\
Henkel $^{\circledR}$ diluído (HD) & 44,968 & 5049,90 \\
\hline
\end{tabular}

Segundo Nock e Richter [24] a viscosidade do PVAc em temperatura ambiente pode chegar a 20.000 cP. Para utilização, a cola deve ser diluída em água para a redução da viscosidade. Quanto maior a viscosidade, maior dificuldade de espalhamento, menor propriedade de umectação, menor penetração do adesivo na estrutura capilar da madeira, resultando numa linha de cola mais espessa com qualidade inferior de colagem. Quanto menor a viscosidade, maior a penetração do adesivo e absorção pela madeira, o que pode resultar numa linha de cola faminta [25]. Para Follrich et al. [13] a penetração do adesivo exerce grande influência no desempenho da ligação.

De acordo com Özçifçi e Yapıcı [22], a viscosidade do PVAc deve ser $16000 \pm 3000 \mathrm{mPa} \cdot \mathrm{s}$, em seu trabalho a viscosidade do adesivo foi de $14000 \pm 3000 \mathrm{mPa} \cdot \mathrm{s}$. Para Iwakiri [21], a viscosidade do PVAc à uma temperatura de $25^{\circ} \mathrm{C}$, varia entre $6000 \mathrm{cP}$ a $8000 \mathrm{cP}$. Bandel [26] afirma que a viscosidade pode variar dependendo da temperatura e do tipo de viscosímetro utilizado.

\subsection{Transformação das amostras em corpos de prova.}

Após a colagem das peças e o tempo de cura já estabelecido, foram confeccionados os corpos de prova para atingir as dimensões estabelecidas pela Norma NBR 7190:1997 [27], e, em seguida, foram realizados os ensaios de cisalhamento na linha de cola. A Figura 1 mostra as etapas da submissão aos testes de ruptura dos corpos de prova. 


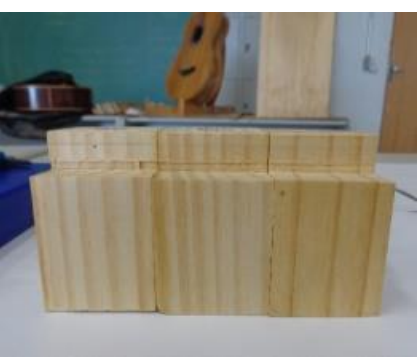

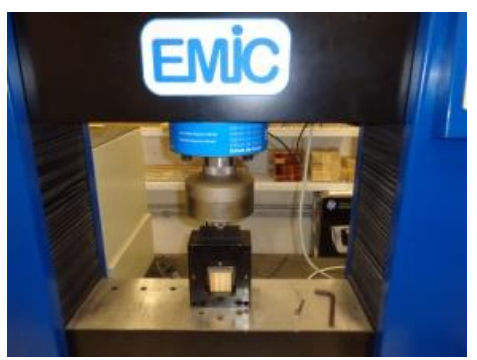

b

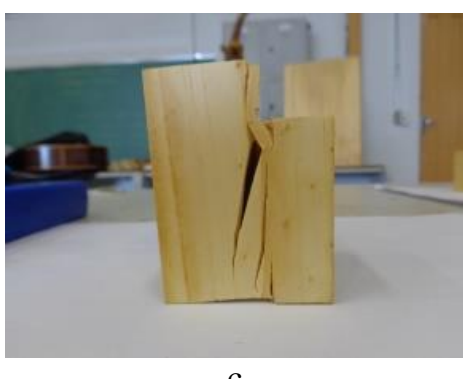

Figura 1: Etapas da submissão aos testes de ruptura dos corpos de prova: a) Corpos de prova preparados para o ensaio de cisalhamento; b) Ensaio de caracterização de resistência mecânica na linha de cola; c) Corpo de prova após o ensaio de cisalhamento.

\subsection{Testes de resistência mecânica}

Para os ensaios de caracterização da resistência ao cisalhamento na ruptura da linha de cola do Pinus, com planos aleatórios do mesmo material, empregou-se uma máquina computadorizada de ensaio universal, marca EMIC, modelo DL 30000, com capacidade para realizar ensaios mecânicos para uma carga de até 30 toneladas, disponível no Laboratório de Propriedades dos Materiais do Campus da UNESP de Itapeva/SP.

Foram preparados e selecionados 15 corpos de prova para cada tipo de adesivo (diluído e não diluído) para realizar os ensaios de resistência, totalizando 60 corpos de prova.

\subsection{Análises estatísticas}

Os resultados foram analisados pelo programa ANOVA, que realiza uma análise de variância e que considera as diferenças entre médias de tratamento em vista de um nível de significância na faixa de 5\%, os testes Kolmogorov-Smirnov (analisa a homogeneidade da amostra) e Bartlett de variância (dados normais) que, em resumo, nos indica a existência significativa de diferenças entre dois ensaios, ou não, em seguida aplicou-se o teste de Tukey, que é responsável por localizar a diferença entre grupos e padronizar esses resultados.

\section{RESULTADOS}

Os resultados médios de resistência no cisalhamento (fv0) na linha de cola dos adesivos diluídos e não diluídos, acompanhados de seus desvios padrão (DP) e coeficientes de variação (CV), estão apresentados na Tabela 2 .

Tabela 2: Resultados médios obtidos no cisalhamento dos adesivos.

\begin{tabular}{llll}
\hline \multicolumn{4}{c}{ CISALHAMENTO } \\
\hline ADESIVOS & $\boldsymbol{f}_{\boldsymbol{v} 0}(\mathbf{M P a})$ & DP & CV (\%) \\
\hline Madeiranit $^{\circledR}$ diluído (MD) & 6,52 & 1,11 & 17,02 \\
Madeiranit $^{\circledR}$ não diluído (MP) & 6,49 & 1,20 & 18,54 \\
Henkel $^{\circledR}$ diluído (HD) & 5,86 & 1,13 & 19,29 \\
Henkel $^{\circledR}$ não diluído (HP) & 5,15 & 1,38 & 26,90 \\
\hline
\end{tabular}

Na Figura 2, visualizam-se os resultados obtidos nos ensaios de cisalhamento na linha de cola com a utilização dos adesivos diluídos e não diluídos. As relações dos resultados na Figura 2 demonstram, em porcentual, a resistência à ruptura entre os a-desivos diluídos e não diluídos das mesmas marcas: HD/HP = $12,1 \% ; \mathrm{MD} / \mathrm{MP}=0,46 \%$, sendo a mais ex-pressiva a relação Henkel® diluído - Henkel@ não diluído igual a $12,1 \%$, ou seja, o adesivo Henkel ${ }^{\circledR}$ diluído proporcionou um aumento de $12,1 \%$ de resistência mecânica na linha de cola, quando solicitados a esforços de cisalhamento, em relação ao adesivo Henkel@ não diluído.

Em relação aos resultados em porcentual entre as diferentes marcas, diluídos e não diluído têm-se os seguintes resultados: $\mathrm{MD} / \mathrm{HD}=10,12 \% ; \mathrm{MD} / \mathrm{HP}=21,01 \% ; \mathrm{MP} / \mathrm{HD}=9,70 \% ; \mathrm{MP} / \mathrm{HP}=20,65 \%$. O resultado mais expressivo entre as diferentes marcas é $\mathrm{MD} / \mathrm{HP}=21,01 \%$, ou seja, o adesivo Madeiranit@ diluído proporcionou um aumento de $21,01 \%$ de resistência mecânica na linha de cola, quando solicitados a esforços de cisalhamento, em relação ao adesivo Henkel@ não diluído. 


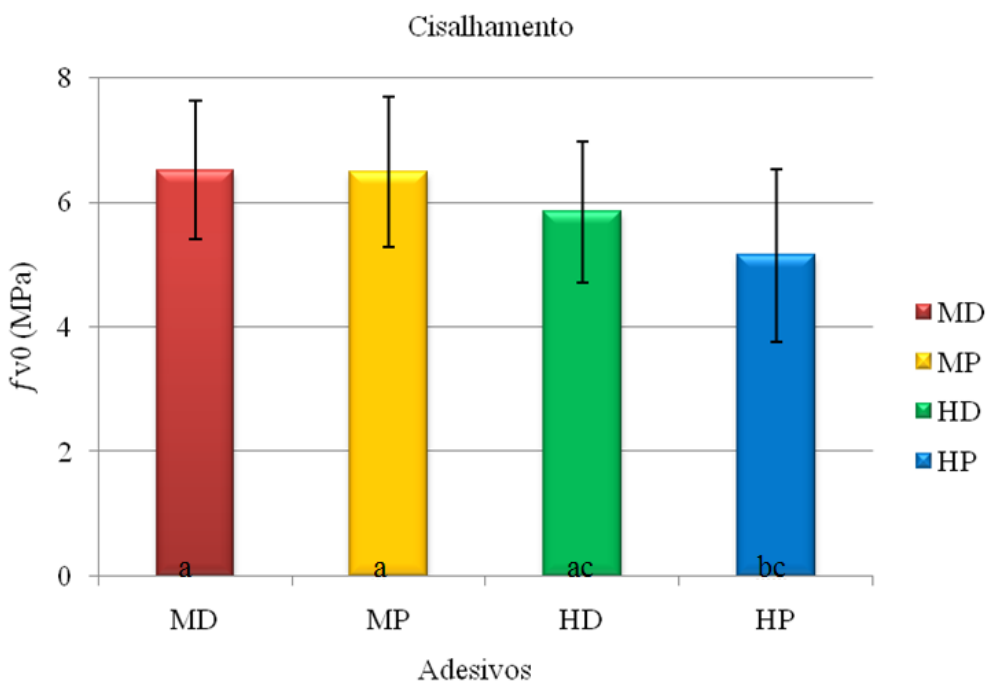

Figura 2: Resultados obtidos nos ensaios de cisalhamento na linha de cola com a utilização dos adesivos diluídos e não diluídos. (*Médias seguidas de mesma letra, não diferem entre si a 5\% de probabilidade pelo teste de Tukey).

\section{DISCUSSÃO}

A madeira de pinus foi usinada nas mesmas condições de usinagem, velocidade de corte $\left(30,18 \mathrm{~m} \cdot \mathrm{s}^{-1}\right)$, velocidade de avanço $\left(11,57 \mathrm{~m} \cdot \mathrm{min}^{-1}\right)$ e avanço por dente $(0,56 \mathrm{~mm})$ para obter a mesma qualidade de acabamento superficial, para a colagem das peças. Silva et al. [28] estudaram as relações entre a anatomia da madeira e a qualidade da usinagem e acabamento da superfície e concluíram que a qualidade final de um produto relaciona-se com as operações de usinagem e com a estrutura física e anatômica da madeira.

Os resultados da Tabela 1 demonstram que o adesivo Madeiranit $\circledast$ diluído apresentou melhor adesão, ou seja, melhor desempenho mecânico, entretanto o adesivo Henkel® não diluído apresentou pior desempenho.

A boa penetração do adesivo na madeira é um aspecto muito importante da colagem de madeira [15], [29]. Se a penetração for profunda aumenta a área de superfície de contato entre adesivo e a madeira, tornando-se mais eficaz a ancoragem mecânica do adesivo [5]. Para que ocorra essa ligação forte com a madeira, o adesivo deve alcançar a camada de madeira intacta abaixo da superfície, penetrando e preenchendo os lúmens, pontuação das células e as fendas da parede celular [12], [30]. Outro fator que poderia justificar o melhor desempenho dos adesivos diluídos está relacionado à viscosidade [24].

Vital et al. [31] avaliando a resistência ao cisalhamento na linha de cola de dois adesivos comerciais de PVAc, de média e alta viscosidade, aplicados a madeira de Pinus elliottii, encontraram valores de tensão de ruptura iguais a 5,41 MPa e 5,64 MPa, respectivamente.

Em comparação aos resultados obtidos, valores encontrados por [32] estão próximos ao obtido neste trabalho, pelo adesivo Henkel ${ }^{\circledR}$ não diluído e diluído, 5,15 MPa e 5,86 MPa, respectivamente, e abaixo dos valores obtidos pelo adesivo Madeiranit ${ }^{\circledR}$ diluído e não diluído. E estão bem abaixo dos valores encontrados no Pinus sylvestris [32] que obtiveram na posição tangencial o valor de 14,52 MPa com o adesivo PVAc.

Lopes et al.[33] obteve para juntas coladas de madeira de Pinus taeda L. com adesivo PVAc, valores médios de resistência ao cisalhamento de 7,21 MPa para faces de colagem tangencial estando próximo aos valores do adesivo Madeiranit $\circledast$ diluído e não diluído, no entanto estando abaixo para faces de colagem radial que é 9,10 MPa.

Os adesivos diluídos apresentaram melhores resultados no cisalhamento na linha de cola em relação aos seus adesivos sem diluir da mesma marca, o que pode ser justificado pelas prováveis ocorrências: maior penetrabilidade na estrutura anatômica da madeira devido à diminuição da viscosidade, melhorando a molhabilidade (umectação, fluidez e penetração) do adesivo na superfície da madeira e, consequentemente, o seu espalhamento, favorecendo a sua penetração propiciando uma linha de cola. Como consequência, a penetração mais profunda aumenta a área de superfície de contato entre adesivo e madeira, tornando mais eficaz a ancoragem mecânica. 


\section{CONCLUSÕES}

Ambos adesivos na forma diluída com 33,33\% de água deionizada proporcionaram uma maior adesão em relação à formulação original, quando solicitados por esforços de cisalhamento na linha de cola. Foi mais expressivo o adesivo do tipo Madeiranit ${ }^{\circledR}$ na forma diluída (MD) que proporcionou um aumento de $21,01 \%$ de resistência mecânica na linha de cola quando solicitado por esforços de cisalhamento em relação ao outro adesivo do tipo $\mathrm{Henkel}^{\circledR}$ em sua formulação original.

Os adesivos Madeiranit, na forma diluída e não diluída, não apresentaram diferencas significativas em relação à resistência à ruptura, entre si, no entanto os adesivos da marca Henkel ${ }^{\circledR}$ na forma diluída e não diluída apresentaram uma diferença de adesão um pouco mais relevante entre si.

\section{BIBLIOGRAFIA}

[1] SEGUNDINHO, P. G. A., GONÇALVES, F. G., GAVA, G. C., et al., "Eficiência da colagem de madeira tratada de Eucalyptus cloeziana F. Muell para produção de madeira laminada colada (MLC)", Revista Matéria, v. 22, n. 2, 2017.

[2] FERREIRA, B. S., CAMPOS, C. I., SILVA, M. S., et al., "Cisalhamento na Linha de Cola de Compensa-dos de Eucalyptus sp. e Adesivo PVA", Floresta e Ambiente, v. 19, n. 2, pp. 141-146, 2012.

[3] SOUZA, M. O. A., SILVA, J. C., DELLA LUCIA, R. M., et al., "Avaliação da Madeira de Eucalyptus camaldulensis Dehnh e Eucalyptus urophylla S.T. Blake em Ensaios de Usinagem, Visando à Produção Moveleira", Revista Árvore, v. 33, n. 4, p. 751-758, 2009.

[4] GONÇALVES, M. T. T., Processamento da Madeira. Bauru-SP, Document Center Xerox - USC, 2000.

[5] FRIHART, C. R., HUNT, C. G., "Adhesives with Wood Materials Bond Formation and Performance", In: Wood Handbook - Wood as an Engineering Material, chapter 10, U.S. Department of Agriculture, Forest Service, Forest Products Laboratory, 2010.

[6] KOLLMANN, F. F. P., KUENZI, E. W., STAMM, A. J., Principles of Wood Science and Technology -II - Wood Based Materials, v. 2, New York. Springer-Verlag, 1975.

[7] KOLLMANN, F. F. P., CÔTÉ, W. A., Principles of Wood Science and Technology - I - Solid Wood, v. 1, New York. Springer-Verlag, 1984.

[8] GALEMBECK, F., GANDUR, M. C., "Cientistas explicam o fenômeno da adesão" Química e Derivados, v. 393, pp. 24-32, 2001.

[9] MANO, E. B., MENDES, L. C., Introdução a polímeros, 2. ed. rev. e ampl., 2004.

[10] PIZZI, A., Advanced wood adhesives technology. New York: Marcel Dekker, 1994.

[11] SCHULTZ, J., NARDIN, M., "Theories and Mechanisms of Adhesion”, In: PIZZI, A., MITTAL, K. L., (eds) Handbook of Adhesive Technology, 2 ed, chapter 3, New York, USA, Marcel Dekker, 2003.

[12] JAKES, J. E., FRIHART, C. R., STONE, D. S., "Nanoindentation size effects in wood”, In: 30th Annual Meeting of The Adhesion Society. pp. 15-17. February 18-21, 2007.

[13] FOLLRICH, J., VAY, O., VEIGEL, S., "Bond strength of end-grain joints and its dependence on surface roughness and adhesive spread", Journal of wood science, v. 56, n. 5, pp. 429-434, 2010.

[14] COOL, J., HERNÁNDEZ, R. E., "Evaluation of four surfacing methods on black spruce wood in relation to poly(vinyl acetate) gluing performance”, Wood and Fiber Science, v. 43, n. 2, pp. 1-12, 2011.

[15] FRIHART, C. R., "Wood adhesion and adhesives", In: ROWELL, R. M.(ed), Handbook of Wood Chemistry and Wood Composites, chapter 9, CRC Press, New York, 2005.

[16] PETRIE, E. M., Handbook of Adhesives and Sealants, McGraw-Hill, New York, 1999.

[17] SALVINI, A., SAIJA, L. M., LUGLI, M., et al., "Synthesis of Modified Poly(vinyl acetate) Adhesives", In: PIZZI, A., MITTAL, K. L. Wood Adhesives, Part 3: Environment-Friendly Adhesives, pp. 317-339, 2010.

[18] SANTOS, C. S. F., Caracterização de Adesivos baseados em Dispersões Aquosas Vinílicas Desenvolvimento de Métodos de Ensaio, Dissertação M.Sc., Universidade Técnica de Lisboa, Lisboa, 2008.

[19] MESQUITA, A. C., Estudo da polimerização do acetato de vinila utilizando a radiação ionizante, Dissertação de M.Sc., IPEN, Autarquia associada à Universidade de São Paulo, São Paulo, 2002. 
[20] KIM, S., KIM, H. J., "Thermal stability and viscoelastic properties of MF/PVAc hybrid resins on the adhesion for engineered flooring in under heating system; ONDOL”. Thermochimica Acta, v. 444, n .2, pp. 134-140, 2006.

[21] IWAKIRI, S., Painéis de madeira reconstituída, FUPEF, Curitiba, PR, 2005.

[22] ÖZÇIFÇI, A., YAPICI, F., "Structural performance of the finger-jointed strength of some wood species with different joint configurations", Construction and Building Materials, v. 22, n. 7, pp. 1543-1550, 2008.

[23] INSTITUTO DE PESQUISAS TECNOLÓGICAS - IPT. Disponível em: <http://www.ipt.br/informacoes_madeiras/60.htm>. Acessado em Novembro de 2016.

[24] NOCK, H. P., RICHTER, H. G., Adesão e Adesivos -Adesivos e sua aplicação na indústria madeireira, Tópicos de Tecnologia da Madeira, 94p. Universidade Federal do Paraná, Curitiba, 1978.

[25] DIAS, F. M. Aplicação de resina poliuretana à base de mamona na fabricação de painéis de madeira compensada e aglomerada, Tese de D.S.c, USP, Universidade de São Paulo, São Carlos, SP, Brasil, 2005.

[26] BANDEL, A. Adhesivos y Tecnologia del Encolado en la Industria de la Madera, Milano, 1991.

[27] ASSOCIAÇÃO BRASILEIRA DE NORMAS TÉCNICAS. NBR-7190: Projeto de estruturas de madeira. Rio de Janeiro, ABNT - Associação Brasileira de Normas Técnicas, 1997.

[28] SILVA, J. R. M., MENDES, L. M., TRUGILHO, P. F., et al., "Preparação de superfícies de madeira e derivados para receber acabamento”, Boletim Agropecuário, Lavras, v. 28, pp.05-26, 1999.

[29] FRIHART, C. R., “Adhesive Bonding and Performance Testing of Bonded Wood Products”, Journal of ASTM International, v. 2, n. 7, July/August, 2005.

[30] ALBUQUERQUE, C. E. C., LATORRACA, J. V. F. "Influência das características anatômicas da madeira na penetração e adesão de adesivos”, Revista Floresta e Ambiente, v. 7, n. 1, pp. 158-166, jan./dez., 2000 .

[31] VITAL, B. R., MACIEL, A. S., DELLA LUCIA, R. M. Qualidade de juntas coladas com lâminas de madeiras de três regiões do tronco de Eucalyptus grandis, Eucalyptus saligna e Pinus elliottii, Revista Árvore, v. 30, n. 4, p. 637-644, 2006.

[32] ÖZÇIFÇI, A., YAPICI, F, "Effects of machining method and grain orientation on the bonding strength of some wood species", Journal of Materials Processing Technology, Amsterdam, v. 202, n. 1/3, pp. 353-358, June 2007.

[33] LOPES, M. C., MUNIZ, G. I. B., MATOS, J. L. M., et al., "Resistência da linha de cola de painéis de Pinus taeda L colados lateralmente com diferentes adesivos”, Cerne, v. 19, n. 4, p. 613-619, out./dez, 2013.

\section{ORCID}

João Carlos Biazzon

Victor Almeida De Araujo

Paulo Roberto Gomes Alves

Elen Aparecida Martines Morales Marcos Tadeu Tibúrcio Gonçalves

Ivaldo De Domenico Valarelli http://orcid.org/0000-0003-2843-9165

http://orcid.org/0000-0002-2747-4738

http://orcid.org/0000-0002-2599-8853

http://orcid.org/0000-0002-9013-1578

http://orcid.org/0000-0001-6414-9179

http://orcid.org/0000-0002-0760-5163 